\title{
Application of (bio) chemical engineering principles and lumping analysis in modelling the living systems
}

\author{
Gheorghe Maria* \\ Department of Chemical \& Biochemical Engineering, University Politehnica of Bucharest, Romania
}

Submission: February 07, 2017; Published: February 10, 2017

*Corresponding author: Gheorghe Maria, Department of Chemical \& Biochemical Engineering, Politehnica University of Bucharest, Splaiul Independenței 313, București 060042, Romania, Email: gmaria99m@hotmail.com

\section{Abstract}

The "whole-cell" simulation of cell metabolic processes under considering a variable-volume modelling framework has been reviewed to prove their advantages when building-up modular model structures of simplified form that can reproduce complex protein syntheses inside cells. The more realistic "whole-cell-variable-volume" (VVWC) approach is reviewed when developing modular kinetic representations of the homeostatic gene expression regulatory modules (GERM) that control the protein synthesis and homeostasis of metabolic processes. The paper review the general concepts of the VVWC modelling, while the cited literature includes past and current experience with GERM linking rules in order to point-out how optimized globally efficient kinetic models for the genetic regulatory circuits (GRC) can be obtained to reproduce experimental observations. Based on quantitative regulatory indices evaluated vs. simulated dynamic and stationary environmental perturbations, the reviewed literature exemplifies with GERM -s from E. coil, at a generic level, how this methodology can be extended:

i) To characterize the module efficiency, species connectivity, and system stability;

ii) To build-up modular regulatory chains of various complexity;

iii)To prove feasibility of the cooperative vs. concurrent construction that ensures an efficient gene expression, system homeostasis, proteic functions, and a balanced cell growth during the cell cycle;

iv) To prove the effect of the whole-cell content ballast in smoothing the effect of internal/external perturbations on the system homeostasis.

Keywords: Kinetic modelling of cell metabolic processes; Homeostatic regulation of gene expression; Regulatory modules of gene expression (GERM); Linking GERM-s

\section{Introduction}

Living cells are organized, self-replicating, self-adjustable, evolvable and responsive structures to environmental stimuli. Attempts to model metabolic cell reactions and processes are not new, an adequate dynamic model being the one engineering alternative to coherently, consistently, and systematically insilico represent the cell metabolism aiming at studying the cell response to various perturbations (reviews $[5,6,8,17,18]$ ). Thus, Synthetic Biology and System Biology become emergent sciences focus on the engineering-driven model-based building of complex biological entities, aiming at applying engineering principles of systems design to biology with the idea to produce predictable and robust biological systems with novel functions in a broad area of applications, such as therapy of diseases (gene therapy), design of new biotechnological processes, new devices based on cell-cell communicators, biosensors, etc. "System
Biology can be defined as "the science of discovering, modelling, understanding and ultimately engineering at the molecular level the dynamic relationships between the biological molecules that define living organisms." (Leroy Hood, President Institute for System Biology, Seattle, USA, cited by $[17,18])$.

Due to the highly complex and partly unknown aspects of the metabolic processes, the detailed mathematical modelling at a molecular level remains still an unsettled issue, even if remarkable progresses and developments of extended simulation platforms have been reported. The general modelling rules, based on physico-chemical-biological and chemical engineering principles, and a statistical data treatment are more difficult to be applied to living systems. That is because metabolic cell processes present a low observability vs. the very large number of species of the order $\mathrm{O}\left(10^{4}\right)$, reactions $\mathrm{O}\left(10^{5}\right)$, 
and transport parameters. Application of advanced lumping techniques can increase the model estimability by reducing the number of reactions and/or variables, and by keeping the most influential terms. Model quality tests, parameter and species sensitivity analysis, principal component and algorithms to find invariant subspaces are common rules to reduce extended model structures. The reduction cost is a loss of information on certain species and reactions, a loss in model generality, prediction capabilities, and physical meaning for some rate constants.

To overcome the structural low identifiability of living cell processes, the current trend is to use all types of information 'translated' from the 'language' of molecular biology to that of mechanistic chemistry, by preserving the cell structural hierarchy and species functions. Application of (bio) chemical engineering concepts and modelling methods, and of the nonlinear systems control theory allow improving the cell model quality, and may offer a detailed simulation of the cell metabolism adaptation to environmental changes, useful for designing modified genetic circuits and of modified micro-organisms.

Several case studies [1,2,6-16] exemplify application of such a gradual lumping analysis and modular approach to derive valuable models able to mimic the stationary and perturbed cell growth, cell response to stimuli, and system homeostasis under isotonic osmolarity.

Applications [5,15-19,45,60,2,8-13,31,39,43,50] refer to insilico design (that is by using mathematical models and tools) of mutant cells with desirable motifs, such as genetic switches acting as biosensors, or genetic circuits amplifying exogeneous stimuli, or involved in signal transduction, or in oscillatory cell processes such as glycolysis. Another case study [31] presents a multi-layer/multi-scale model that couples a structured representation of a metabolic genetic regulatory circuit at a molecular level with the macroscopic mass balances of the relevant state variables of a fluidized-bed bioreactor used for mercury uptake from wastewaters by using immobilized E. coil bacteria. The model was proved useful for process design and optimal control purposes allowing predicting the wild/cloned bacteria metabolism adaptation (i.e. the response of the italics letters operon expression) over several cell generations to dynamic operating conditions of the bioreactor.

The present paper is aiming at reviewing the general concepts of the VVWC modelling approach when developing modular kinetic representations of the homeostatic gene expression regulatory modules (GERM) that control the protein synthesis and homeostasis of metabolic processes. The paper is also reviewing some published contributions including past and current experience with GERM linking rules in order to point-out how optimized globally efficient kinetic models for the genetic regulatory circuits (GRC) can be obtained to reproduce experimental observations.

\section{Applied Concepts When Modelling the GRC Dynamics}

It is well-known that most of the concepts and numerical methods used in chemical engineering can be also used when modelling the dynamics of enzymatic [40-42,20-36] and metabolic cell processes [1,2,6-16]. The reviewed simple case studies of VVWC modular kinetic models of GERM-s [6-16] (see Figure 1 for such a reduced representation of a GERM) proved that the chemical and biochemical engineering principles, together with the control theory of the nonlinear systems are fully applicable to modelling complex metabolic cell processes, including the sophisticated GRC-s controlling the cell enzymes syntheses and metabolic fluxes [52].

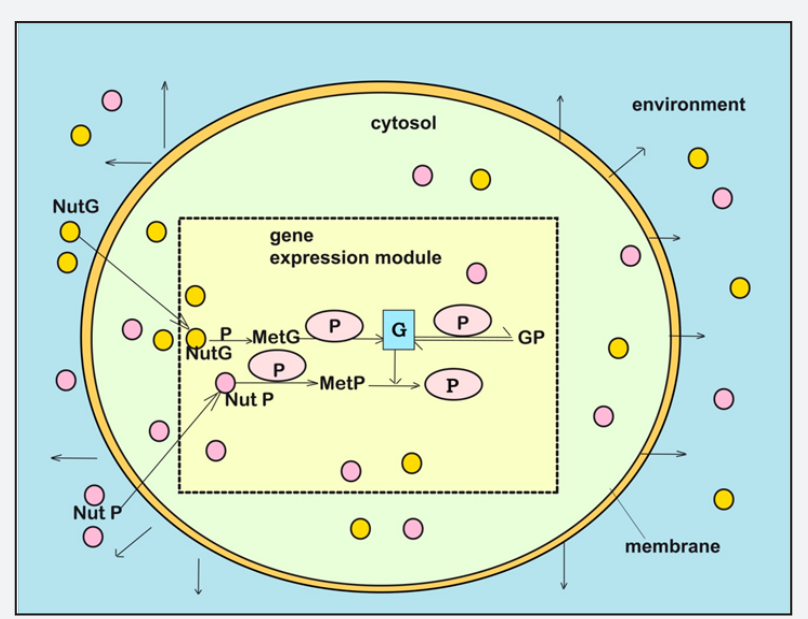

Figure 1: The reaction scheme of a generic gene $G$ expression. The regulatory module of $G(P) 1$ type was used to exemplify the synthesis of a generic $\mathrm{P}$ protein in the $E$. coil cell by Maria [22]. To improve the system homeostasis stability, that is quasi-invariance of key species

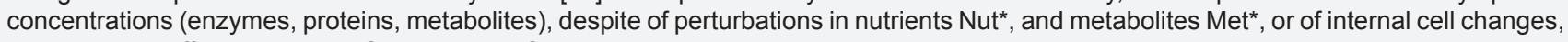
a very rapid buffering reaction $\mathrm{G}+\mathrm{P}<===>\mathrm{GP}$ (inactive) has been added. Horizontal arrows indicate reactions; vertical arrows indicate catalytic actions; $\mathrm{G}$ = gene encoding protein $\mathrm{P} ;$ MetG, MetP = lumped DNA and protein precursor metabolites respectively. 


\section{These Principles and Tools Includes}

i) Molecular species conservation law (stoichiometry analysis; species differential mass balance set);

ii) Atomic species conservation law (atomic species mass balance);

iii) Thermodynamic analysis of reactions (quantitative assignment of reaction directionality), [3]; set equilibrium reactions; Gibbs free energy balance analysis; set cyclic reactions; find species at quasi-steady-state; improved evaluation of steady-state flux distributions that provide important information for metabolic engineering and on cell metabolism [4];

iv) Application of species and/or reaction lumping rules to the Ordinary Differential Equation ODE model $[1,38]$;

v) Analysis of cyclic reactions, and of the cell system steady-state (homeostasis) stability. Such kinetic analysis at a molecular level includes modelling the kinetics of complex GRC-s [1,2,6-16] controlling protein synthsis and cell metabolic fluxes distribution, and resource allocation in branched pathways through genetic switches according to environmental conditions [1,2,6-13,39,43,50]. Such a cell model can eventually be used to design modified micro-organisms. Deterministic (model-based) simulation platforms allow italics letters design of modified cells with desirable gene circuits and 'motifs' of practical applications in the biosynthesis industry, environmental engineering, and medicine [13-19,5,45,60], or for modelling and design of new drug delivery systems with a controlled drug release $[15,37,44,46,49,55-57]$.

Some of these applications have been developed, for instance, for modelling the gene expression regulation [1,2,616]. Due to near astronomic complexity of cell processes (see Figure 2 as an example of central carbon metabolism [51,54]), and a huge number of involved species of order $\mathrm{O}\left(10^{4}\right)$, and reactions $\mathrm{O}\left(10^{5}\right)$, advanced lumping procedures [38,56] and modularization techniques $[1,6,8,10,12,13,31]$ have been applied to obtain reduced models by lumpig species and/or reactions but keeping the main cell functions, and the structural, functional and temporal hierarchy.

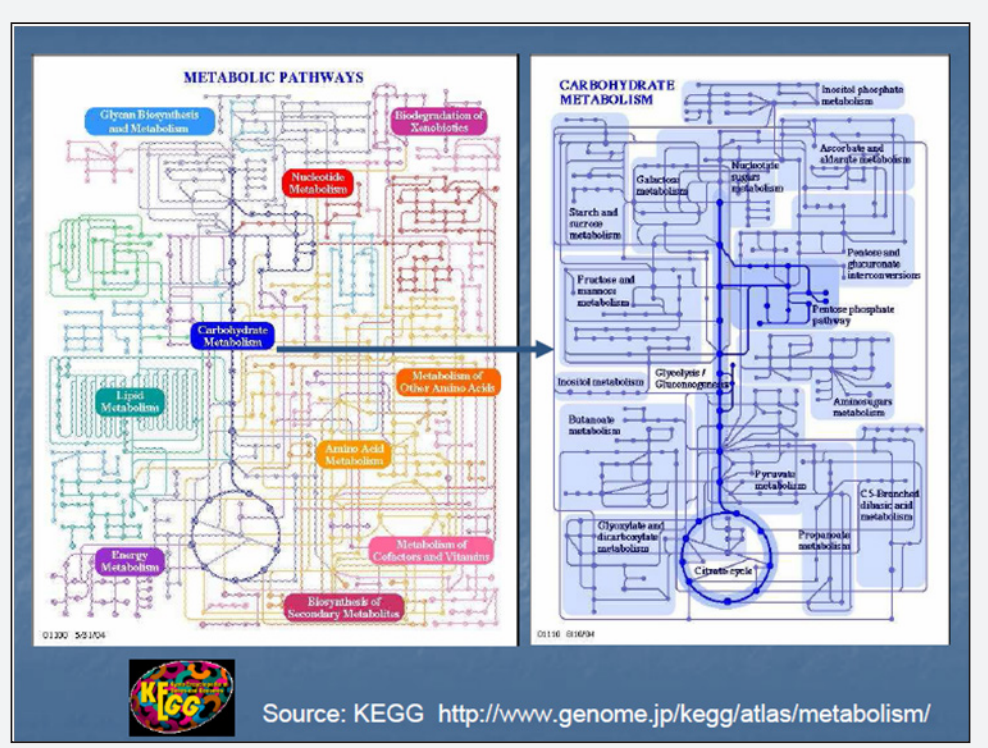

Figure 2: The central carbon metabolism pathway in E. coli (from KEGG -omic free databank).

When developing whole-cell models on a mechanistic (deterministic) basis by using continuous-variable models, all enzymatic processes are closely linked to ensure an optimum metabolism. So, the metabolic reactions must occur with maximum reaction rates, with using minimum of resources (substrates, energy), and producing minimum amount of reaction intermediates; besides, reactions and key-species homeostasis should be less influenced by the environmental perturbations by involving simple GRC-s with a preferable cascade control of the gene expression that minimize the transition or recovering times of the quasi-steady-states QSS $[7,43]$. It quickly appeared that lot of cell continuous processes can be modelled by using the concepts of the (bio) chemical kinetic modelling. For simplicity, the modelling problem was decomposed in modelling „functional modules" which, eventually will be linked to recreate the wholecell structure $[15,16]$ that is "modules that can be elaborated .....by 'translating' from the 'language' of molecular biology to that of mechanistic chemistry, by preserving the cell structural hierarchy and component functions" [1]. Other attempts to model cell complex continuous processes to reproduce the three main properties of the cell metabolism: i) dynamics; ii) feedback, and iii)optimality, by using „Electronic circuits” like models [1] (Figure 3) failed because they cannot reproduce in detail molecular interactions with slow and continuous responses to perturbations. 


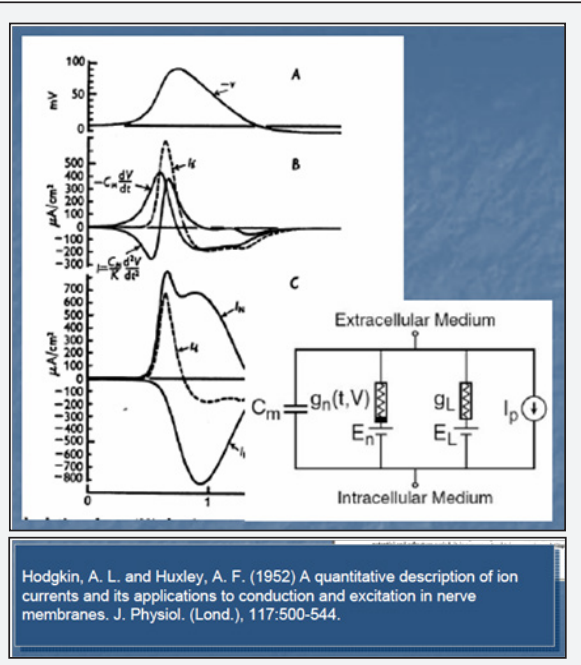

Figure 3: Attempt to model oscillatory cell processes with electronic-like circuit models.

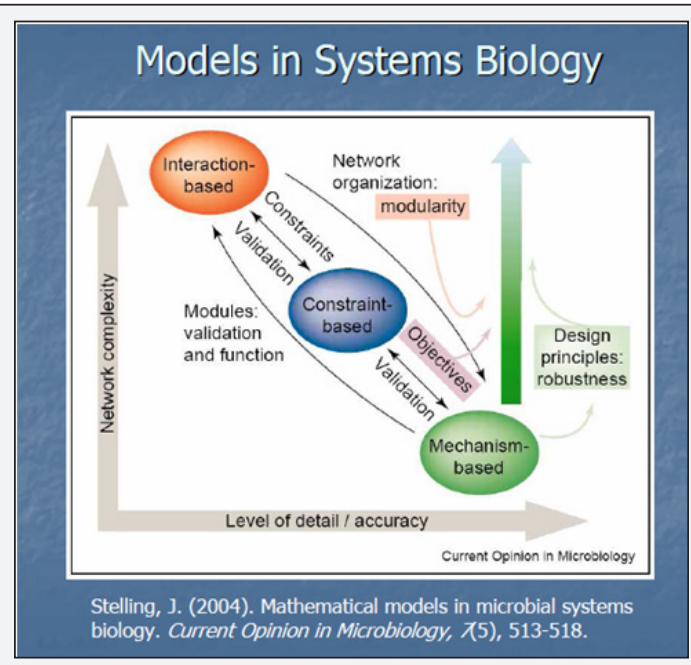

Figure 4: The increase of the deterministic model complexity with the level of detail.

As expected, because the deterministic model complexity sharply increases with the level of detail (Figure 4), a satisfactory model complexity that realizes the best trade-off between model simplicity and its predictive quality should be adopted [7]. When developing a structured dynamic model with continuous variables, there are strong reasons to use lumping techniques for reducing its complexity. And that is because: i) there are too complex cell mechanisms vs. available data; ii) there is a large number of species, reactions, transport parameters, and interactions difficult to be modelled in detail; iii) structured experimental data (standard kinetic data) are difficult to be obtained and usually present a low observability and reproducibility due to the metabolic process variability; iv) a reduced cell process model allows an easier interpretation of cell complexity, and quick simulations of cell behavior under various environmental conditions. Besides, the computational tractability of the reduced mathematical models allows application of well-known algorithmic rules of chemical engineering, nonlinear system theory, and numerical calculus.
Inherently, the reduced dynamic cell models suffer from a series of drawbacks,

such as:

i. Multiple reduced structures of different characteristics are possible to exist for the same cell process, difficult to be discriminated;

iii. Loss of information on certain species and reaction steps;

iv. Loss in system flexibility, due to the reduced number of intermediates and species interactions included in the mathematical model;

v. Loss in prediction capabilities;

vi. Lumped model parameters can lack of physical meaning;

vii. Loss/alteration of systemic/holistic properties 
(stability, multiplicity, sensitivity, regulatory characteristics).

Starting from some case studies, elaborated procedures have been proposed to buildup a whole-cell simulator of modular construction [1,2,7-14], useful for evaluation of the genetic regulatory circuits' (GRC) efficiency, and in designing genetically modified cells with desirable characteristics („motifs”). Thus, there are a large number of important contributions in modelling regulatory cell processes. Among the pioneers, are to be mentioned: Heinrich and Schuster, [52]; Athel Cornish Bowden [53], Torres and Voit [58], or construction of modular GRC-s $[19,38,39,45,47,53,60]$ to mention only few of them. It is also to notice that the number of papers in the area of System Biology increases with 3-orders of magnitude in the last 10 -years, according to Scopus.

Basically, the main hypotheses of a VVWC model are the following [1]:

(i) The cell system consists in a sum of hierarchically organized components, e.g. metabolites, genes DNA, proteins, RNA, intermediates, etc. (interrelated through transcription, translation, and DNA replication and other processes); the cell is separated from the environment (containing nutrients) by a membrane.

(ii) The membrane, of negligible volume, presents a negligible resistance to nutrient diffusion; the membrane dynamics being neglected in the cell model, is assumed to follow the cell growing dynamics.

(iii) The cell is an isothermal system with an uniform content (perfectly-mixed case); species behave ideally, and present uniform concentrations within cell. The cell system is not only homogeneous but also isotonic (constant osmotic pressure), with no inner gradients or species diffusion resistance.

(iv) The cell is an open system interacting with the environment through a semi-permeable membrane.

(v) To better reproduce the GERM properties interconnected with the rest of the cell, the other cell species are lumped together in the so-called "cell ballast". To fulfil the adopted Pfeiffers'law of diluted solutions:

$$
V(t)=\frac{R T}{\pi_{c y t}} \sum_{j=1}^{n_{S}} n_{j}(t)
$$

[where $\mathrm{T}$ = absolute temperature; $\mathrm{R}$ = universal gas constant, $\mathrm{V}=$ cell (cytosol) volume; $\pi_{\text {cyt }}=$ inner osmotic pressure; $\mathrm{t}=$ time; $\mathrm{n}_{\mathrm{j}}=$ species $\mathrm{j}$ number of moles], Maria [1,2,6-13] proposed that lumped genome and proteome replication to also be considered in such cell models. Concentration $=\frac{\text { no. of copies } / \text { cell }}{N_{A} \times V_{\text {cyt,o }}}$

(vii) The inner osmotic pressure $\left(\pi_{\text {cyt }}\right)$ is constant, and all time equal with the environmental pressure, thus ensuring the membrane integrity $\left(\pi_{\text {cyt }}=\pi_{\text {env }}=\right.$ constant $)$. As a consequence, the isotonic osmolarity under isothermal conditions leads to the equality RT $/ \pi_{\text {cyt }}=\mathrm{RT} / \pi_{\text {env }}$ which, indicating that the sum of cell species concentrations must equal those of the environment, i.e. $\left(\sum_{j}^{a l l} C_{j}\right)_{c y t}=\left(\sum_{j}^{a l l} C_{j}\right)_{e n v}$. Otherwise, the osmosis will eventually lead to an equal osmotic pressure $=\pi_{\text {cyt }}=\pi_{\text {env }}$. Even if, in a real cell, such equality is approximately fulfilled due to perturbations and transport gradients, and in spite of migrating nutrients from environment into the cell, the overall environment concentration is considered to remain unchanged. On the other hand, species inside the cell transform the nutrients into metabolites and react to make more cell components. In turn, increased amounts of polymerases are then used to import increasing amounts of nutrients. The net result is an exponential increase of cellular components in time, which translates, through isotonic osmolarity assumption, into an exponential increase in volume with time [1]. The overall concentration of cellular components is time-invariant (homeostasis), because the rate at which cell-volume increases equals that at which overall number of moles increases, leading to a constant $\left(\sum_{j=1}^{n_{s}} n_{j}\right) / V$ ratio.

(viii) The species concentrations $\mathrm{Cj}=\mathrm{nj} / \mathrm{V}$ at the cell level are usually expressed in nano-molar, being computed with the relationship [1]:

When modelling metabolic cell processes with deterministic continuous-variable models by using a certain number of novel concepts related to the VVWC modelling approach, certain advantages have to be underlined, as followings $[1,2,6-15,60]$

The more realistic "whole-cell-variable-volume" (VVWC) approach which was proved to lead to a more effective representation of cell processes, being very useful when developing modular kinetic representations of the GERM-s that control the protein synthesis and homeostasis of metabolic processes.

The novel VVWC modelling framework to buildup kinetic cell models presents the advantage of explicitely including in the model equations the isotonicity constraint, and the variable cell volume link with the cell reactions. The holistic approach reveals the role played by the "cell ballast" in smoothing the effect of perturbations coming from the environment. The VVWC modelling framework was succesfully used to derive effective kinetic models describing various genetic regulatory circuits (GRC), and individual gene expression modules (GERM) [1,2,616].

The VVWC model formulation was proved to be also suitable to accurately model the cell growth and its division [59]. Such a model formulation allows studying various regulatory properties of GERM-s, and the response of coupled GERM-s to dynamic / stationary continuous perturbations in the environment, and also the 'inertial' effect of the cell- 'ballast' vs. continuous changes in cell and environment [1,2,6-13]. As ca. $80 \%$ of the cycle period is the growing phase and, assuming a quasi-constant osmotic pressure and a constant volume growing logarithmic rate, the VVWC cell model can be considered satisfactory to study the GRC effectiveness. 
The novel VVWC modelling framework allows a realistic analysis of rules to be used for GERM linking when buiding-up complex GRC dynamic models, thus offering the possibility

i) To simulate the regulatory performances of a gene expression, of an aperon expression, or

ii) Of a GRC (switch, amplifier, filter, etc. $[39,43,50])$, and also

iii) To in-silico design of genetic modified or cloned micro-organisms with target plasmides to get desirable characteristics for industrial or medical applications. Some examples include: maximization of succinate production in E. coli [60]; efficient removal of mercury from wastewaters [10,12,29,31,34]; design of a genetic switch of desirable characteristics $[2,9,13]$.
The novel VVWC modelling framework was used to simulate some cell metabolic processes by using the concept of a modular simulation platform, extensible, and linked to -omic databanks (Ecocyc, KEGG, Brenda, Prodoric, CellML, NIH, etc. $[1,13,14,31,51,54])$. By using conventional and non-conventional estimation techniques and incomplete experimental data from literature, applications have reproduced the protein synthesis homeostatic regulation, or efficiency of some GRC [1,2,6-13]. Such reduced dynamic deterministic models can fairly simulate some essential cell processes, such as glycolysis in Escherichia coli cells (Figure 5), under stationary or perturbed growing conditions. Such a modular cell simulator can be used to insilico design genetic modified or cloned micro-organisms with industrial or medical applications [1,2,6-15,60].

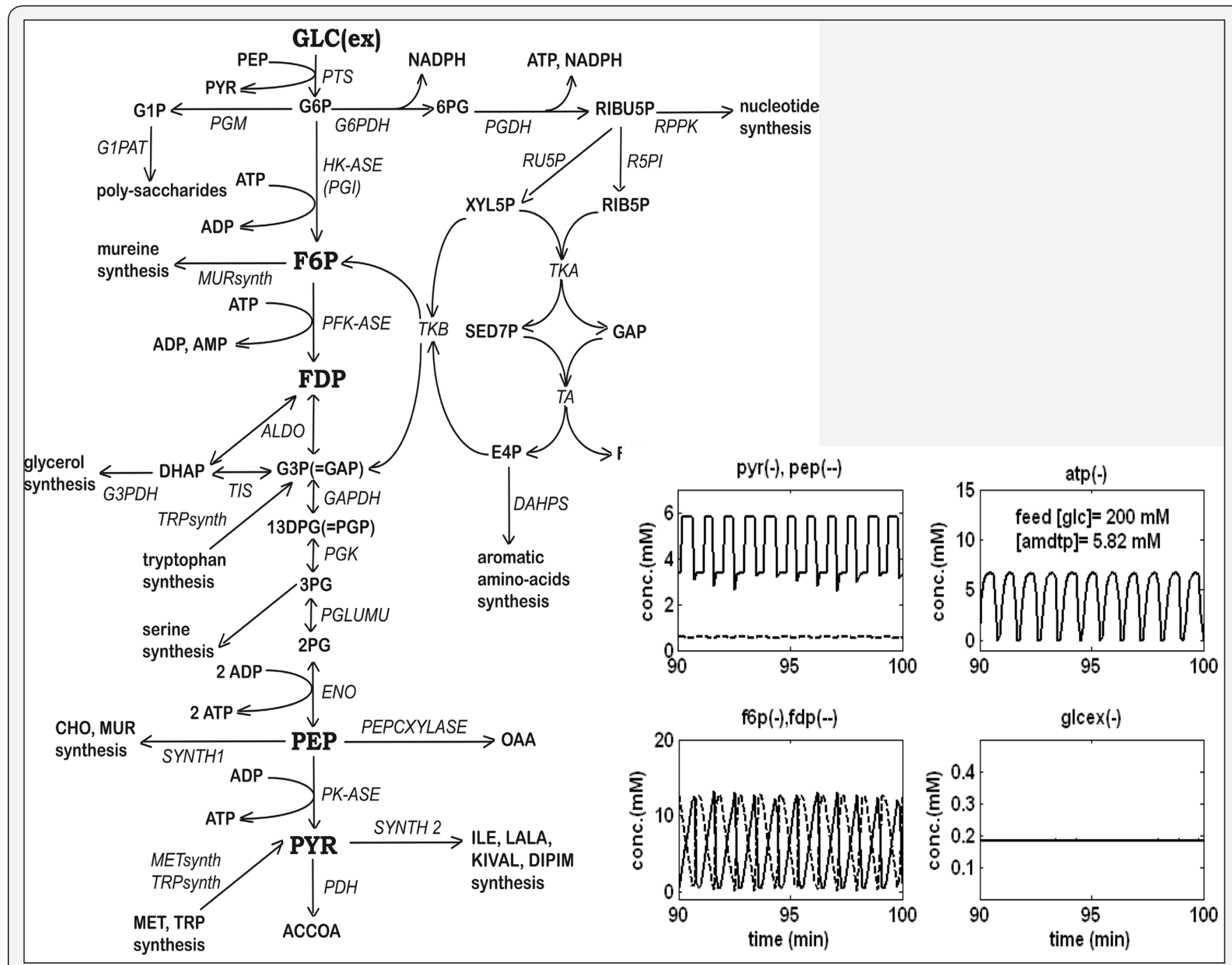

Figure 5: The structured reduced model of Maria [14] used to simulate the oscillating glycolysis in Escherichia coli. 


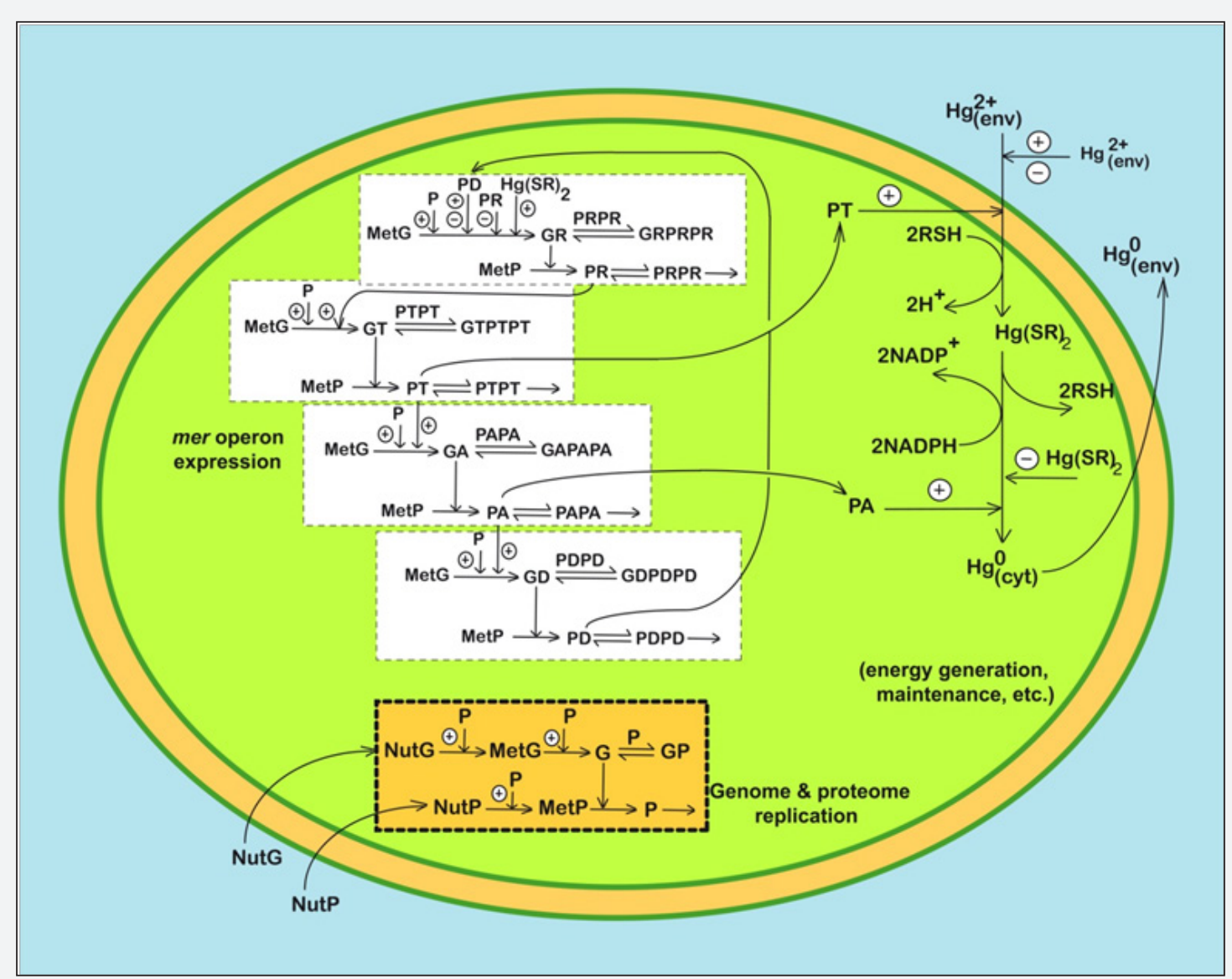

Figure 6: Structured cell model of Maria [12,31] used to simulate the dynamics of mer-operon expression for mercury uptake in Escherichia coli.

The mechanistic approach was very adequate when developing a structured complex kinetic model (Figure 6) to simulate the efficiency of the GRC responsible for induced expression of the mer-operon in gram-negative bacteria (Pseudomonas putida, E. coli) responsible for regulation of the mercury ions uptake from wastewaters [10,12,29,31,34]. The model was tested in the new VVWC modelling framework vs. literature data and used to optimize operation of an industrial fluidized-bed bioreactor used for mercury removal from wastewaters [31,34].

\section{Conclusion}

As revealed by this very brief review, general chemical engineering modelling principles are proved to be valuable tools for representing the both stationary and dynamic characteristics of complex cell biochemical processes. Elaboration of reduced models of satisfactory quality is closely related to the ability of selecting the suitable lumping rules, key-parameters, and influential terms, and to apply multi-objective non-/conventional estimation criteria that realize the best trade-off between model simplicity and its predictive quality.

Note: This paper is the extended version of the invited plenary lecture:
Maria, G., Applications of Chemical Engineering Principles and the Lumping Analysis in Modelling the Living Systems - A trade-off between simplicity and model quality, presented at University Babes-Bolyai, Cluj (Romania), Department of Chemistry, Nov. 8, 2013. http://www.chem.ubbcluj.ro/ chimie/ anunturi.html,

And of the plenary lecture: Maria, G., Applications of Chemical Engineering Principles and the Lumping Analysis in Modelling the Living Systems -A trade-off between simplicity and model quality, Symposium SICHEM-2016, University Politehnica of Bucharest, 8 Sept. 2016.

\section{References}

1. Maria G (2005) Modular-Based Modelling of Protein Synthesis Regulation, Chemical and Biochemical Engineering Quaterly 19(3): 213-233.

2. Maria G (2007) Modelling bistable genetic regulatory circuits under variable volume framework, Chemical and Biochemical Engineering Quarterly 21(4): 417-434.

3. Haraldsdottir HS, Thiele I, Fleming RMT (2012) Quantitative assignment of reaction directionality in a multicompartmental human metabolic reconstruction. Biophys J 102(8): 1703-1711.

4. Zhu Y, Song J, Xu Z, Sun J, Zhang Y, et al (2013) Development of thermodynamic optimum searching (TOS) to improve the prediction 
accuracy of flux balance analysis. Biotechnology and Bioengineering 110(3): 914-923.

5. Heinemann M, Panke S (2006) Synthetic Biology - putting engineering into biology. Bioinformatics 22(22): 2790-2799

6. Maria G (2003) Evaluation of Protein Regulatory Kinetics Schemes in Perturbed Cell Growth Environments by Using Sensitivity Methods. Chemical and Biochemical Engineering Quarterly 17(2): 99-117.

7. Maria G (2006) Application of lumping analysis in modelling the living systems -A trade-off between simplicity and model quality. Chemical and Biochemical Engineering Quarterly 20(4): 353-373.

8. Maria G (2008) Reduced modular representations applied to simulate some genetic regulatory circuits. Revista de Chimie 59(3): 318-324.

9. Maria G (2009) Lumped dynamic model for a bistable genetic regulatory circuit within a variable-volume whole-cell modelling framework. Asia-Pacific Journal of Chemical Engineering 4(6): 916928.

10. Maria G (2009) A whole-cell model to simulate the mercuric ion reduction by $\mathrm{E}$. coli under stationary and perturbed conditions. Chemical and Biochemical Engineering Quarterly 23 (3): 323-341.

11. Maria G (2008) A whole-cell approach in modelling genetic circuits in living cells. New Biotechnology 25: S338.

12. Maria, G (2010) A dynamic model to simulate the genetic regulatory circuit controlling the mercury ion uptake by E. coli cells. Revista de Chimie 61(2): 172-186

13. Maria G (2014) Extended repression mechanisms in modelling bistable genetic switches of adjustable characteristics within a variable cell volume modelling framework. Chemical \& Biochemical Engineering Quarterly 28(1): 35-51.

14. Maria G (2015) Insilico derivation of a reduced kinetic model for stationary or oscillating glycolysis in Escherichia coli bacterium. Chemical \& Biochemical Engineering Quarterly 28(4): 509-529.

15. Hudder B, Yang Q, Bolfing B, Maria G, Morgan JJ, et al. (2002) Computational Modeling of Iron Metabolism in Mitochondria, NIST Conference on 'Systems Biology Approaches to Health Care: Mitochondrial Proteomics, Gaithersburg, USA, p. 1.

16. Visser D, Schmid J.W, Mauc, K, Reuss M, Heijnen J.J(2004) Optimal redesign of primarymetabolism in Escherichia coli using linlog kinetics. Metabolic Engineering 6(4): 378-390.

17. Banga JR (2008) Optimization in computational systems biology, $6^{\text {th }}$ Simon Stevin on Optimization in Engineering, Arenberg, Leuven, NL.

18. Banga JR (2008) Optimization in computational systems biology. BMC Systems Biology 2: 47.

19. Rodriguez-Fernandez M, Mendes P, Banga JR (2006) A hybrid approach for efficient and robust parameter estimation in biochemical pathways. BioSystems 83(2-3): 248-265.

20. Maria G, Rippin DWT (1997) Modified Integral Procedure (MIP) as a Reliable Short-Cut Method for Kinetic Model Estimation: Isothermal, Non-Isothermal and (Semi-) Batch Process Cases. Computers \& Chemical Engineering 21(10): 1169-1190.

21. Maria G, Maria C, Salcedo R, Feyo de Azevedo S (2000) Databank Transfer-of-Information, Shortcut and Exact Estimators Used in the Wastewater Biological Treatment Process Identification. Computers \& Chemical Engineering 24(2-7): 1713-1718.

22. Treitz G, Maria G, Giffhorn F, Heinzle E (2001) Kinetic Model Discrimination via step-by-step Experimental and Computational Procedure in the Enzymatic Oxidation of D-Glucose. Jl Biotechnology 85(3): 271-287.
23. Maria G (2007) Model-Based Heuristic Optimised Operating Policies for D-Glucose Oxidation in a Batch Reactor with Pulsate Addition of Enzyme. Computers \& Chemical Engineering 31(10): 1231-1241.

24. Maria G, Cocuz A (2011) Operating alternatives for the (semi)batch reactor used for D-glucose enzymatic oxidation with free-enzyme, Revista de Chimie 62(3): 318-327.

25. Maria G (2012) Enzymatic reactor selection and derivation of the optimal operation policy by using a model-based modular simulation platform, Computers \& Chemical Engineering 36(1): 325-341.

26. Maria G, Ene M.D, Jipa I (2012) Modelling enzymatic oxidation of D-glucose with pyranose 2-oxidase in the presence of catalase. Journal of Molecular Catalysis B: Enzymatic 74(3-4): 209-218.

27. Ene M.D, Maria G (2012) Temperature decrease (30-25oC) influence on bi-enzymatic kinetics of D-glucose oxidation. Journal of Molecular Catalysis B: Enzymatic 81(9): 1924

28. Maria G, Ene, MD (2012) Kinetics studies on enzymatic oxidation of d-glucose with pyranose 2-oxidase in the presence of catalase. New Biotechnology 29(23-26): S86.

29. Maria G, Luta I, Maria C (2013) Model-based sensitivity analysis of a fluidised-bed bioreactor for mercury uptake by immobilised Pseudomonas putida cells. Chemical Papers 67(11): 1364-1375.

30. Maria G, Ene M.D (2013) Modelling enzymatic reduction of 2-ketoD-glucose by suspended aldose reductase. Chemical \& Biochemical Engineering Quarterly 27(4): 385-395.

31. Maria G, Luta I (2013) Structured cell simulator coupled with a fluidized bed bioreactor model to predict the adaptive mercury uptake by E. coli cells. Computers \& Chemical Engineering 58:98-115.

32. Maria G, Crisan, M (2015) Evaluation of optimal operation alternatives of reactors used for D-glucose oxidation in a bi-enzymatic system with a complex deactivation kinetics. Asia-Pacific Journal of Chemical Engineering 10(1): 22-44.

33. Crisan M, Maria G (2016) Modular simulation to check performances of various reactors for the enzymatic D-glucose oxidation. Revue Roumaine de Chimie (in-press) 61(6-7): 549-556.

34. Scoban A.G, Maria G (2016) Model-based optimization of the feeding policy of a fluidized bed bioreactor for mercury uptake by immobilized P. putida cells. Asia-Pacific Journal of Chemical Engineering 11(5): 721 734.

35. Maria G (2016) Quick identification of a simple enzyme deactivation model for an extended-Michaelis-Menten reaction type. Exemplification for the D-glucose oxidation with a complex enzyme deactivation kinetics. Computers \& Chemical Engineering 93(4): 323-330.

36. Crisan M, Maria G (2017) Modular simulation to determine the optima operating policy of a batch reactor for the enzymatic fructose reduction to mannitol with the in-situ continuous enzymatic regeneration of the NADH cofactor, Revista de Chimie, accepted.

37. Luta I, Maria G (2012) In-silico modulation of the irinotecan release from a functionalized MCM-41 support. Chemical \& Biochemical Engineering Quarterly 26(4): 309-320.

38. Maria GA (2004) Review of Algorithms and Trends in Kinetic Model Identification for Chemical and Biochemical Systems. Chemical and Biochemical Engineering Quarterly 18(3): 195-222.

39. Savageau MA (2002) Alternatives designs for a genetic switch: Analysis of switching times using the piecewise power-law representation. Math. Biosciences 180: 237-253.

40. Maria G, Ognean T (1989) An Adaptive Parameter Estimation used to Obtain Reduced Kinetic Models for the Biological Treatment Process. Water Research 23(2): 175-181. 
41. Maria G, Rippin D.W.T (1995) Modified Integral Procedure (MIP) as a Reliable Short-Cut Method in Mechanistical Based ODE Kinetic Model Estimation: Non-Isothermal and Semi-Batch Process Cases. Computers \& Chemical Engineering 19(Suppl 1): 709-S714.

42. Maria G, Rippin DWT(1996) Recursive Robust Kinetics Estimation by Using Mechanistic Short-Cut Technique and a Pattern-Recognition Procedure, Computers \& Chemical Engineering 2: S587-S592.

43. Savageau MA, Voit EO (1987) Recasting nonlinear differential equations as S-systems: A canonical nonlinear form. Math. Biosciences 87: 83-115.

44. Maria G, Stoica AI, Luta I, Stirbet D, Radu GL (2012) Cephalosporin release from functionalized MCM-41 supports interpreted by various models. Microporous and Mesoporous Materials 162(11): 80-90.

45. Moles CG, Mendes P, Banga JR (2003) Parameter estimation in biochemical pathways: A comparison of global optimization methods. Genome Res 13(11): 2467-2474.

46. Maria G, Berger D, Nastase S, Luta I (2012) Modelling alternatives of the irinotecan release from functionalized mesoporous-silica supports, Microporous and Mesoporous Materials 149(1): 25-35.

47. Sauro HM, Kholodenko, BN (2004) Quantitative analysis of signalling networks. Prog Biophys Mol Biol 86(1): 5-43.

48. Kholodenko BN, Kiyatkin A, Bruggeman FJ, Sontag E, Westerhoff HV, et al. (2002) Untangling the wires: A strategy to trace functional interactions in signalling and gene networks. Proc Natl Acad Sci USA 99(20): 12841-12846

49. Maria G, Luta I (2015) Kinetic models for the in-silico design of functionakized mesoporous supports for the controlled release of biological active principles (Eds,). Printech, Bucharest, Romania, pp.476.

50. Wall ME, Hlavacek WS, Savageau MA (2003) Design principles for regulator gene expression in a repressible gene circuit. J Mol Biol 332(4): 861-876.

51. Tomita M, Hashimoto K, Takahashi K, Shimizu TS, Matsuzaki Y, et al (1999) E-Cell: Software environment for whole cell simulation Bioinformatics 15(1): 72-84.

52. Heinrich R, Schuster S (1996) The regulation of cellular systems Chapman \& Hall, London, UK.

53. Athel Cornish Bowden (2004) The Pursuit of Perfection: Aspects of Biochemical Evolution,Oxford University Press, USA.

54. Tomita M (2001) Whole-cell simulation: a grand challenge of the 21st century Trends in Biotechnology 19(6): 205-210.

55.Zhang W, Tichy SE, Pérez LM, Maria GC, Lindahl PA, et al. (2003) Evaluation of Multivalent Dendrimers Based on Melamine. Kinetics of Dithiothreitol - Mediated Thiol-Disulfide Exchange Depends on the Structure of the Dendrimer. Journal of American Chemical Society 125(17): 5086-5094.

56. Maria G (2005) Relations between Apparent and Intrinsic Kinetics of Programmable Drug Release in Human Plasma. Chemical Engineering Science 60(6): 1709-1723.

57. Maria G, Luta I (2011) Precautions in using global kinetic and thermodynamic models for characterization of drug release from multivalent supports. Chemical Papers 65(4): 542-552.

58. Torres NV (2002) Pathway analysis and optimization in metabolic engineering, Cambridge University Press, Cambridge, USA.

59. Morgan JJ, Surovtsev IV, Lindahl PA (2004) A framework for whole-cell mathematical modelling J Theor Biol 231(4): 581-596.

60. [60] Maria, G., Xu, Z., Sun, J., Investigating alternatives to in-silico find optimal fluxes and theoretical gene knockout strategies for E. coli cell, Chemical \& Biochemical Engineering Quarterly 25(4), 403-424 (2011).

\section{Your next submission with Juniper Publishers will reach you the below assets}

- Quality Editorial service

- Swift Peer Review

- Reprints availability

- E-prints Service

- Manuscript Podcast for convenient understanding

- Global attainment for your research

- Manuscript accessibility in different formats

( Pdf, E-pub, Full Text, Audio)

- Unceasing customer service

Track the below URL for one-step submission https://juniperpublishers.com/online-submission.php 